\title{
Three-body correlations in Borromean halo nuclei
}

F. M. Marqués ${ }^{1, *}$ M. Labiche, ${ }^{1, \dagger}$ N. A. Orr, ${ }^{1}$ J. C. Angélique, ${ }^{1}$ L. Axelsson, ${ }^{2}$ B. Benoit,${ }^{3}$ U. C. Bergmann, ${ }^{4}$ M. J. G. Borge,${ }^{5}$ W. N. Catford, ${ }^{6}$ S. P. G. Chappell, ${ }^{7}$ N. M. Clarke, ${ }^{8}$ G. Costa, ${ }^{9}$ N. Curtis, ${ }^{6}+{ }^{\circ}$ A. D’Arrigo, ${ }^{3}$ E. de Góes Brennand, ${ }^{3}$ F. de Oliveira Santos,${ }^{10}$ O. Dorvaux,${ }^{9}$ G. Fazio, ${ }^{11}$ M. Freer,,${ }^{8,1}$ B. R. Fulton, ${ }^{8}$ G. Giardina, ${ }^{11}$ S. Grévy, ${ }^{12,}$ D. Guillemaud-Mueller, ${ }^{12}$ F. Hanappe ${ }^{3}$ B. Heusch, ${ }^{9}$ B. Jonson, ${ }^{2}$ C. Le Brun,,${ }^{1, \|}$ S. Leenhardt, ${ }^{12}$ M. Lewitowicz, ${ }^{10}$ M. J. López, ${ }^{10, \pi}$ K. Markenroth, ${ }^{2}$ A. C. Mueller, ${ }^{12}$ T. Nilsson, ${ }^{2, * *}$ A. Ninane, ${ }^{1, \dagger \dagger}$ G. Nyman, ${ }^{2}$ I. Piqueras, ${ }^{5}$ K. Riisager, M. G. Saint Laurent,${ }^{10}$ F. Sarazin, ${ }^{10, \$}$ S. M. Singer, ${ }^{8}$ O. Sorlin,${ }^{12}$ and L. Stuttgé ${ }^{9}$

${ }^{1}$ Laboratoire de Physique Corpusculaire, IN2P3-CNRS, ISMRA et Université de Caen, F-14050 Caen cedex, France

${ }^{2}$ Experimentell Fysik, Chalmers Tekniska Högskola, S-412 96 Göteborg, Sweden

${ }^{3}$ Université Libre de Bruxelles, CP 226, B-1050 Bruxelles, Belgium

${ }^{4}$ Institut for Fysik og Astronomi, Aarhus Universitet, DK-8000 Aarhus C, Denmark

${ }^{5}$ Instituto de Estructura de la Materia, CSIC, E-28006 Madrid, Spain

${ }^{6}$ Department of Physics, University of Surrey, Guildford, Surrey GU2 7XH, United Kingdom

${ }^{7}$ Department of Nuclear Physics, University of Oxford, Keble Road, Oxford OX1 3RH, United Kingdom

${ }^{8}$ School of Physics and Astronomy, University of Birmingham, Birmingham B15 2TT, United Kingdom

${ }^{9}$ Institut de Recherche Subatomique, IN2P3-CNRS, Université Louis Pasteur, BP 28, F-67037 Strasbourg cedex, France

${ }^{10}$ GANIL, CEA/DSM-CNRS/IN2P3, BP 55027, F-14076 Caen cedex, France

${ }^{11}$ Dipartimento di Fisica, Università di Messina, Salita Sperone 31, I-98166 Messina, Italy

${ }^{12}$ Institut de Physique Nucléaire, IN2P3-CNRS, F-91406 Orsay cedex, France

(Received 10 January 2001; revised manuscript received 30 March 2001; published 5 November 2001)

Three-body correlations in the dissociation of two-neutron halo nuclei are explored using a technique based on intensity interferometry and Dalitz plots. This approach provides for the combined treatment of both the $n-n$ and core- $n$ interactions in the exit channel. As an example, the breakup of ${ }^{14} \mathrm{Be}$ into ${ }^{12} \mathrm{Be}+n+n$ by $\mathrm{Pb}$ and $\mathrm{C}$ targets has been analyzed and the halo $n-n$ separation extracted. Evidence for a finite delay between the emission of the neutrons in the reaction on the $\mathrm{C}$ target was observed and is attributed to ${ }^{13} \mathrm{Be}$ resonances populated in sequential breakup.

DOI: 10.1103/PhysRevC.64.061301

PACS number(s): 25.10.+s, 21.10.Gv, 21.45.+v, 27.20.+n

The quest for the drip lines, which define the limits of binding for nuclear systems, has only been attained for light nuclei. As such, these nuclei are unique in displaying the manner in which nucleons bind in an $A$ nucleon system from the most neutron deficient to the most neutron rich. Clustering phenomena, observed for example in excited states close to threshold [1], also appear as neutron haloes in ground states near the neutron drip line [2]. Perhaps the most intriguing manifestations of clustering are the Borromean twoneutron halo nuclei $\left({ }^{6} \mathrm{He},{ }^{11} \mathrm{Li}\right.$, and $\left.{ }^{14} \mathrm{Be}\right)$, in which the twobody subsystems are unbound [3]. Such behavior naturally gives rise to the question of the correlations between the constituents of these three-body systems. Even in the case of the most studied of these nuclei, ${ }^{6} \mathrm{He}$ and ${ }^{11} \mathrm{Li}[4-8]$, little is known in this respect.

The dissociation in the field of a target nucleus, followed by the measurement of the momenta of the fragments (core

\footnotetext{
*Email address: Marques@caelav.in2p3.fr

${ }^{\dagger}$ Present address: University of Paisley, Scotland.

${ }^{\ddagger}$ Present address: University of Birmingham, U.K.

${ }^{\S}$ Present address: LPC, Caen, France.

${ }^{\|}$Present address: ISN, Grenoble, France.

${ }^{\mathbb{T}}$ Present address: CENBG, Bordeaux, France.

**Present address: ISOLDE, CERN, Switzerland.

${ }^{\dagger}$ On leave from UCL, Louvain-la-Neuve, Belgium.

\# Present address: University of Edinburgh, Scotland.
}

$+n+n)$, has been used in attempts to probe correlations in two-neutron halo nuclei. Vestiges, however, of the two-body forces that stabilize the projectile in the ground state may affect the three-particle decay in the form of final-state interactions (FSI). Experimentally, beyond the reconstruction of the core $+2 n$ invariant mass, the analyses so far reported have been restricted to the binary channels. In the case of $n-n$ observables, which should in principle provide access to the correlations within the halo, comparison has typically been made to simplified interpretations such as a dineutron configuration or three-body phase-space decay [5,9], neglecting the $n-n$ FSI. In the core- $n$ channel, reactions induced by a light target have been used to probe the formation of resonances following the removal of one of the halo neutrons [5-7]. The reconstruction of the momentum of the removed neutron can, as described in Refs. [4,8], provide some insight into the $n-n$ correlations.

In the present Rapid Communication the three-body correlations in the dissociation of two-neutron halo nuclei are explored. In particular, a new method for analyzing triple coincidence events ( core $+n+n)$ from kinematically complete experiments is described. The method incorporates the techniques of intensity interferometry [10] and Dalitz plots [11] and permits the halo $n-n$ separation and time delay between the emission of the two neutrons to be derived. As will be seen, the latter is related to the presence of core- $n$ FSI in the exit channel. In principle, the present approach also al- 


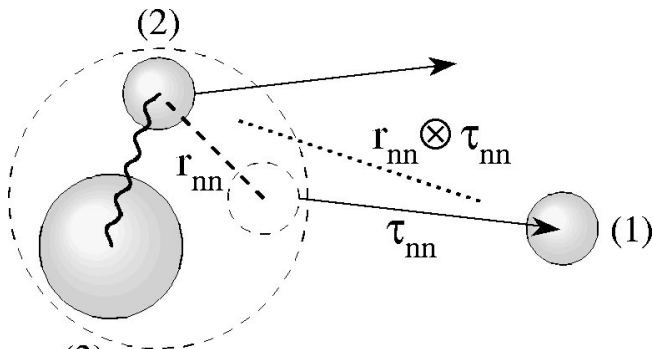

(3)

FIG. 1. Schematic view of the sequential breakup of a twoneutron halo nucleus, whereby dissociation passes through a resonance in the core- $n$ system. A delay $\tau_{n n}$, equivalent to the lifetime of the resonance, is introduced in the emission of the second neutron. The effective source size is a convolution of the $n-n$ separation $r_{n n}$ and the delay $\tau_{n n}$.

lows the energies and lifetimes of these resonances (or virtual states if $l=0$ ) to be derived.

When neutrons are emitted in close proximity in spacetime, the wave function of relative motion is modified by the known FSI and quantum statistical symmetries [10]. Twoneutron intensity interferometry, and in particular the correlation function [12] $C_{n n}$, relates this modification to the space-time separation of the particles at emission as a function of the four-momenta of the particles:

$$
C_{n n}\left(p_{1}, p_{2}\right)=\frac{d^{2} n / d p_{1} d p_{2}}{\left(d n / d p_{1}\right)\left(d n / d p_{2}\right)} .
$$

The numerator is the measured two-particle distribution and the denominator the product of the independent singleparticle distributions $[12,13]$, which are commonly projected onto one dimension, the relative momentum $q=\left|\vec{p}_{1}-\vec{p}_{2}\right|$. In an earlier paper [13], the correlation function was extracted for the dissociation of ${ }^{14} \mathrm{Be}$ by $\mathrm{Pb}$ with the aim of probing the spatial configuration of the halo neutrons. The analysis followed the formalism of Ref. [14] and assumed that the neutrons were emitted simultaneously following dissociation in the Coulomb field of the target. A $n-n$ separation of $r_{n n}^{\mathrm{rms}}$ $=5.6 \pm 1.0 \mathrm{fm}$ was thus obtained.

The same analysis has been applied to dissociation of ${ }^{14} \mathrm{Be}$ by a $\mathrm{C}$ target, in order to investigate the influence of the reaction mechanism [15]. A result suggestive of a somewhat larger separation, $r_{n n}^{\mathrm{rms}}=7.6 \pm 1.7 \mathrm{fm}$, was obtained. This raises the question as to whether simultaneous emission can be assumed a priori. In principle, the analysis of the correlation function in two dimensions, transverse and parallel to the total momentum of the pair, would allow for the unfolding of the source size and lifetime [14]. Such an analysis requires a large data set and was thus not applicable to the present measurements. The system being studied here is far less complex, however, than those usually encountered in interferometry (for example compound nuclei evaporating particles or systems of colliding heavy ions [10]). Moreover, the simple three-body nature of the system breaking up suggests immediately that any delay in the emission of one of the neutrons will arise from core- $n$ FSI/resonances in the exit channel (Fig. 1). As will be seen later, the degree to which such resonances are present may depend on the reaction mechanism.

The data examined here were acquired from the dissociation of a $35 \mathrm{MeV} /$ nucleon ${ }^{14} \mathrm{Be}$ beam into ${ }^{12} \mathrm{Be}+n+n$ by $\mathrm{Pb}$ and $\mathrm{C}$ targets. Details of the experiment and previous analyses have been reported elsewhere $[13,16]$. Each event was reconstructed from the momenta $\vec{p}_{1,2}$ of the neutrons measured using the DEMON array [17] as follows: (i) we calculate the average velocity $\langle\beta\rangle$ of the core $+n+n$ frame at dissociation as that for which the mean for all events of the total neutron momentum along the beam axis $\left\langle\vec{p}_{1}+\vec{p}_{2}\right\rangle_{z}$ is 0 and the average decay energy of the system $\left\langle E_{d}\right\rangle$ (see below) is a minimum; (ii) in this frame, momentum conservation is applied event-by-event to reconstruct the core momentum $\vec{p}_{3}=-\left(\vec{p}_{1}+\vec{p}_{2}\right)$. From the four-momenta $p_{i}$ of the three particles we calculate the total energy available in the center of mass of the system and extract the kinetic part, the decay energy

$$
E_{d}=\sqrt{\left(\sum_{i=1}^{3} p_{i}\right)^{2}}-\sum_{i=1}^{3} m_{i} .
$$

Note that the present analysis, in which the decay has been reconstructed from only the neutron momenta $\vec{p}_{1,2}$, is similar to that used in Refs. $[4,8]$, whereby the momentum of the removed neutron was reconstructed from the momenta of the core and remaining neutron. The use of this algorithm instead of the more classical analysis employing the core momentum $\vec{p}_{3}$ measured in the charged-particle telescope eliminates the limited energy and position resolution of this detector and the uncertainty related to the depth within the target at which the reaction took place. Importantly, the form of the spectra obtained here for the decay energy of ${ }^{14} \mathrm{Be}$ and the core- $n$ system $\left({ }^{13} \mathrm{Be}\right)$ agree well with those obtained using the core momentum $[16,18]$.

An interacting phase-space model has been developed for the analysis of triple correlations in the data. In brief, the experimental decay energy distribution is used as input to generate events $\vec{p}_{1,2,3}\left(E_{d}\right)$ following three-body phase space [19]. The core- $n$ resonances are introduced following the sequential breakup of the system (Fig. 1) into one neutron and the core- $n$ resonance with a relative energy $E_{23}$ given by a Breit-Wigner distribution $\left(E_{0}, \Gamma\right)$; the resonance is then allowed to decay into the core plus neutron. In the $n-n$ channel, the FSI is introduced via a probability $P\left(\left|\vec{p}_{1}-\vec{p}_{2}\right|\right)$ to accept the event following the form of the measured $n-n$ correlation function [13-15]. The final momenta $\vec{p}_{1,2,3}$ are filtered through a simulation, which includes the range of projectile velocities arising from the finite target thickness and all experimental effects $[13,17]$, and the reconstruction algorithm described above is applied to $\vec{p}_{1,2}$.

Correlations in three-particle decays have been extensively studied in particle physics by means of Dalitz plots of the particle energies $\left(E_{i}, E_{j}\right)$ or the squared invariant masses of particle pairs $\left(M_{i j}^{2}, M_{i k}^{2}\right)$, with $M_{i j}^{2}=\left(p_{i}+p_{j}\right)^{2}$. In these representations, FSI/resonances lead to a nonuniform population of the surface within the kinematic boundary defined 


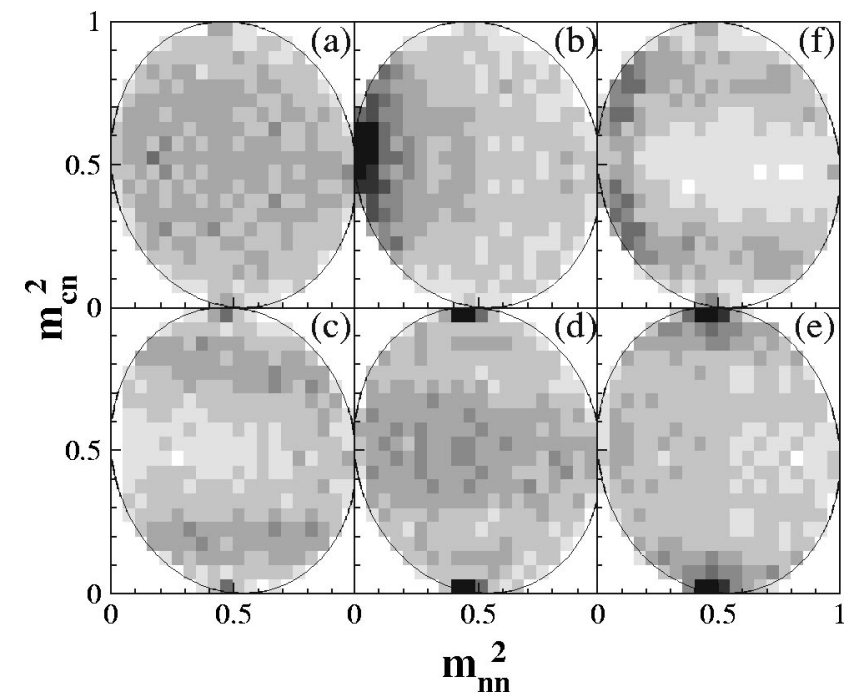

FIG. 2. Dalitz plots (core- $n$ vs $n-n$ ) for simulations with the interacting phase-space model of ${ }^{14} \mathrm{Be}$ dissociation: without FSI (a), with $n-n$ FSI (b), and with a core- $n$ resonance at $E_{0}=0.8$ (c), 2.0 (d), and $3.5 \mathrm{MeV}$ (e). The combination of the $n-n$ and the core- $n$ FSI of (c) is shown in (f).

by energy-momentum conservation and the decay energy [11]. The classic example of such an analysis is the threebody decay of an unstable particle [20]. In the present case, the core $+n+n$ system exhibits a distribution of decay energies. Consequently, the value of $E_{d}$ associated with each event will lead to a different boundary for the Dalitz plot, and the resulting plot containing all events cannot be easily interpreted. We have thus introduced a normalized invariant mass

$$
m_{i j}^{2}=\frac{M_{i j}^{2}-\left(m_{i}+m_{j}\right)^{2}}{\left(m_{i}+m_{j}+E_{d}\right)^{2}-\left(m_{i}+m_{j}\right)^{2}},
$$

which ranges between 0 and 1 (a relative energy $E_{i j}=M_{i j}$ $-m_{i}-m_{j}$ between 0 and $E_{d}$ ) for all events and exhibits a single kinematic boundary. Examples of how $n-n$ and core- $n$ FSI present in the decay are manifested in core- $n$ versus $n-n$ Dalitz plots are displayed in Fig. 2, whereby events have been simulated with the model described above. The inputs were an $E_{d}$ distribution following that measured (Fig. 3), the $C_{n n}$ obtained with the C target [15] (Fig. 4), and core- $n$ resonances with $\Gamma=0.3 \mathrm{MeV}$ at $E_{0}=0.8,2.0,3.5 \mathrm{MeV}$.

In the absence of any FSI, the Dalitz plot exhibits, as noted above, a uniform population [Fig. 2(a)]. The $n-n$ FSI appears as a concentration of events with $m_{n n}^{2} \leq 0.25$ [Fig. 2(b)], which correspond to small relative momenta $[13,15]$. The core- $n$ resonance at $E_{0}=0.8 \mathrm{MeV}$ [Fig. 2(c)] appears as horizontal bands around $m_{c n}^{2} \approx 0.25$ and 0.75 . The location of these bands depends on the energy of the resonance with respect to the mean decay energy of the system: a single band at $m_{c n}^{2} \approx 0.5$ if $E_{0} \sim\left\langle E_{d}\right\rangle$ [Fig. 2(d)] and two symmetric bands if $E_{0} \gtrless\left\langle E_{d}\right\rangle$ [Figs. 2(c), 2(e)] [15]. This feature arises as, when one of the neutrons forms a resonance with the core at a given value of $m_{23}^{2}$, the relative core- $n$ energy of the
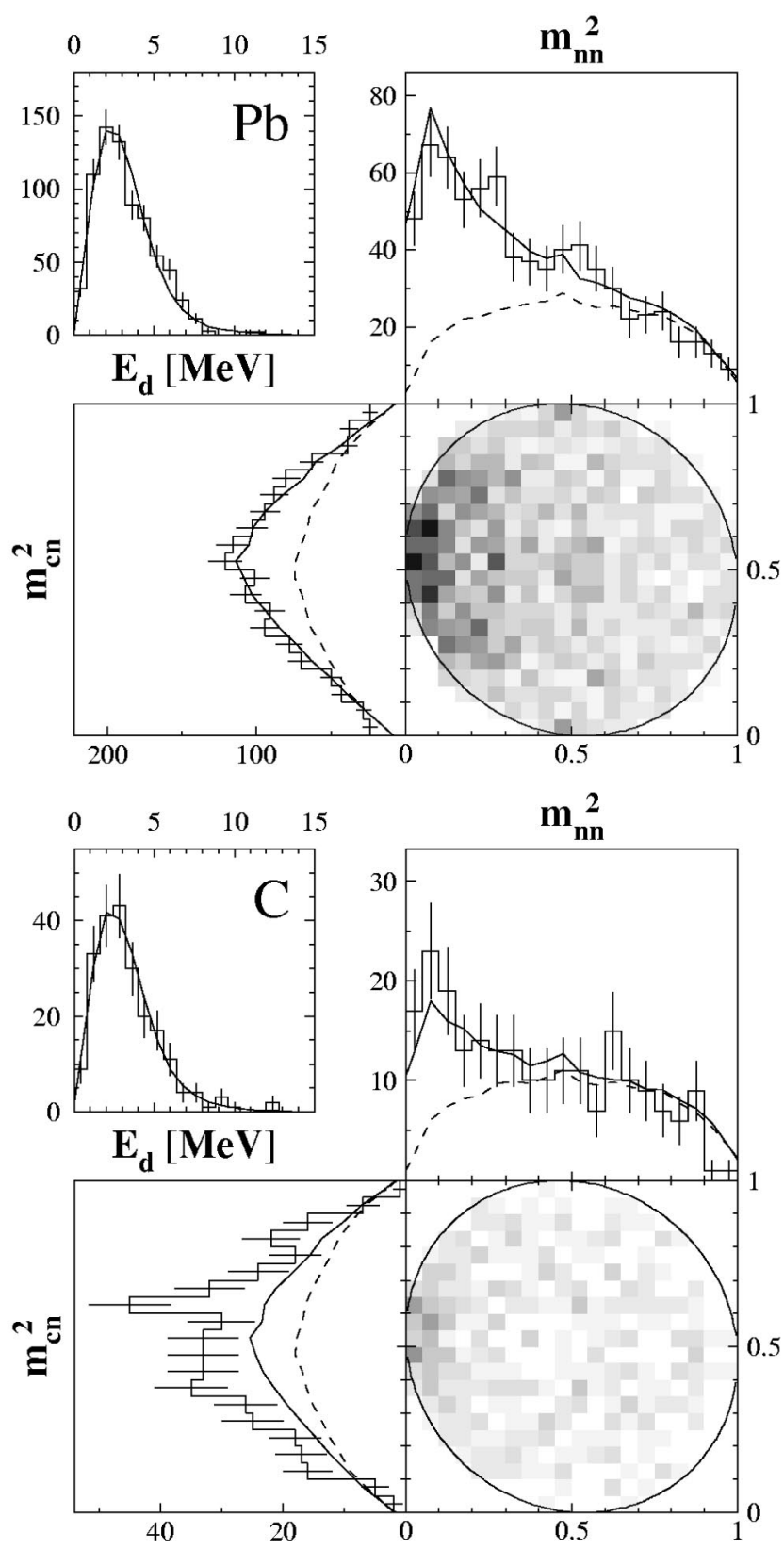

FIG. 3. Dalitz plots (core- $n$ vs $n-n$ ), and the projections onto both axes, for the data from the dissociation of ${ }^{14} \mathrm{Be}$ by $\mathrm{Pb}$ (upper panels) and C (lower panels). The lines are the results of the phasespace model simulations with/without (solid/dashed) $n-n$ FSI. The insets correspond to the ${ }^{14} \mathrm{Be}$ decay energy.

other is essentially fixed at $m_{13}^{2} \approx 1-m_{23}^{2}$ [15]. Importantly, the Dalitz plot representation provides not only for the identification of the different FSI between the particle pairs, but also a direct comparison of the relative importance of each in the decay [Fig. 2(f)].

The Dalitz plot for the data from the dissociation by $\mathrm{Pb}$ (Fig. 3) presents a strong $n-n$ FSI and a uniform density for $m_{n n}^{2} \geq 0.5$. Indeed, the $n-n$ FSI alone describes very well the projections onto both axes, and therefore suggests that core$n$ resonances are not present to any significant extent. This result confirms the hypothesis of simultaneous $n-n$ emission 

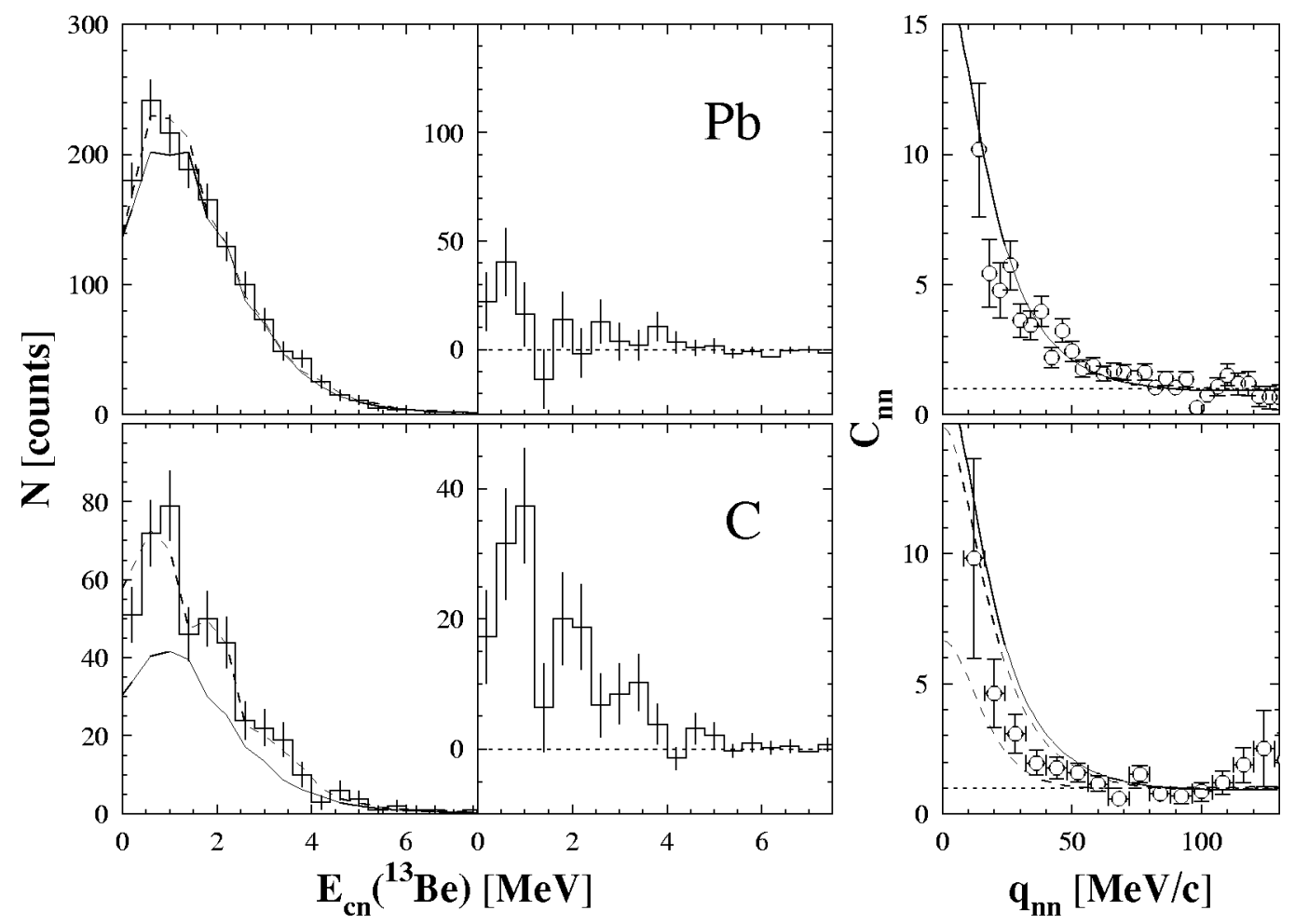

FIG. 4. Core- $n$ relative energy distributions and $n-n$ correlation functions for the dissociation of ${ }^{14} \mathrm{Be}$ by $\mathrm{Pb}$ (upper panels) and $\mathrm{C}$ (lower panels). The lines in the $E_{c n}$ spectra (left) are the result of the phase-space model simulations with $n$ - $n$ FSI (solid lines) plus core- $n$ resonances (dashed lines, see text). The histograms presented in the middle panels are the difference between the data and the $n-n$ FSI simulations. The solid lines in the right panels are the $C_{n n}$ for $r_{n n}^{\mathrm{rms}}=5.6 \mathrm{fm}$ and $\tau_{n n}=0$; the dashed lines correspond to the limits $r_{n n}^{\mathrm{rms}}$ $=6.6-4.6 \mathrm{fm}$ and $\tau_{n n}=0-400 \mathrm{fm} / c$.

employed in the original analysis of the dissociation of ${ }^{14} \mathrm{Be}$ by $\mathrm{Pb}$ [13]. The value of $r_{n n}^{\mathrm{rms}}$ so extracted, $5.6 \pm 1.0 \mathrm{fm}$, thus corresponds to the $n-n$ separation in the halo of ${ }^{14} \mathrm{Be}$.

For dissociation by the $\mathrm{C}$ target (Fig. 3), despite the lower statistics, two differences are evident. First, the $n-n$ signal is weaker, indicating, as discussed earlier, that a significant delay has occurred between the emission of each neutron. Second, and more importantly, the agreement between the model including only the $n-n$ FSI and the data for $m_{c n}^{2}$ is rather poor. In order to verify whether this disagreement corresponds to the presence of core- $n$ resonances, which would be responsible for the weakening of the $n-n$ signal, we have investigated the core- $n$ relative energy, $E_{c n}$. It has been reconstructed for the simulations incorporating only the $n-n$ FSI and compared in Fig. 4 to the data (the model calculations have been normalized to the data above $4 \mathrm{MeV}$ ). For dissociation by $\mathrm{Pb}$, the inclusion of only the $n-n$ FSI provides a very good description of the data, with the exception of small deviations below $1 \mathrm{MeV}$. This is in line with the Dalitz plot analysis discussed above.

The deviations observed for the $\mathrm{C}$ target between the measured $m_{c n}^{2}$ and the simulation including only the $n-n$ FSI (Fig. 3) clearly correspond to structures in the $E_{c n}$ spectrum. Moreover, these structures are located at energies that are in line with those of states previously reported in ${ }^{13} \mathrm{Be}$ : the supposed $d_{5 / 2}$ resonance at $2.0 \mathrm{MeV}$ [21] and a lower-lying state(s) $[18,21,22]$, also suggested by various theoretical calculations [23-26]. The model-to-data ratio is about $1 / 2$, in- dicating that the peaks correspond to resonances formed by one of the neutrons in almost all decays; the solid line in Fig. 4 accounts for the contribution of the neutron not interacting with the core.

If we add to the phase-space model with $n$ - $n$ FSI core- $n$ resonances $(\Gamma=0.3 \mathrm{MeV})$ for all events at $E_{0}=0.8,2.0$ [21] and $3.5 \mathrm{MeV}^{1}$ with intensities of 45,35 , and $20 \%$, respectively, the data are well reproduced (dashed line of Fig. 4). In the case of dissociation by $\mathrm{Pb}$, the lowest-lying resonance(s) appears to be present in at most $10 \%$ of events. This result is in agreement with the analysis presented in Ref. [16], where some $35 \%$ of the two-neutron removal cross section on the $\mathrm{Pb}$ target was attributed to nuclear-induced breakup. The requirement of the detection of two neutrons in coincidence with the ${ }^{12} \mathrm{Be}$ core in the present analysis reduces this to some $15 \%$-approximately half of the two-neutron removal cross section arises from absorption.

The different results obtained for the $\mathrm{Pb}$ and $\mathrm{C}$ targets may be attributed to the associated reaction mechanisms [27]. In the case of the $\mathrm{Pb}$ target, the dominant process is electromagnetic dissociation [16], whereby the halo neutrons behave as spectators and only the charged core is acted on by the Coulomb field of the target [28]. Qualitatively then, the

\footnotetext{
${ }^{1}$ The present data are not particularly sensitive to the location and form of the states, in particular below $1 \mathrm{MeV}$, and a resonance at 0.5 $\mathrm{MeV}$ would, for example, equally well describe the data.
} 
$n-n$ FSI may be expected to influence most strongly the decay. In the case of the $\mathrm{C}$ target, nuclear breakup dominates and the reaction takes place at smaller impact parameters, in general through the interaction of one of the halo neutrons with the target [16]. As such the population of core- $n$ resonances is favored $[6,7]$.

By combining the information extracted from the core- $n$ channel with the $n-n$ correlation functions, we can extend the analysis and also extract the average lifetime of the core- $n$ resonances. If we fix the $n-n$ distance in ${ }^{14} \mathrm{Be}$ as that obtained for dissociation by $\mathrm{Pb}, r_{n n}^{\mathrm{rms}}=5.6 \pm 1.0 \mathrm{fm}$, we can introduce the delay between the emission of the neutrons $\tau_{n n}$ needed to describe the $n-n$ correlation function for the $\mathrm{C}$ target. As discussed earlier, this delay should correspond to the lifetime of the resonances (Fig. 1). The result of a $\chi^{2}$ analysis, represented by the dashed lines in Fig. 4, suggests an average lifetime of $150_{-150}^{+250} \mathrm{fm} / c$, or $\left(5_{-5}^{+8}\right) \times 10^{-22} \mathrm{~s}$.

In summary, three-body correlations in the dissociation of two-neutron halo nuclei have been explored. A new analysis technique employing intensity interferometry and Dalitz plots has been presented and applied to the breakup of ${ }^{14} \mathrm{Be}$ by $\mathrm{Pb}$ and $\mathrm{C}$ targets. Through the combined treatment of both the $n-n$ and core- $n$ correlations, the halo $n-n$ separation has been extracted and a finite delay found between the emission of the neutrons for the reaction on $\mathrm{C}$. This delay can be attributed to resonances in ${ }^{13} \mathrm{Be}$ populated in sequential breakup. The application of the techniques presented here to a well-established system such as ${ }^{6} \mathrm{He}$ would be of particular interest, as would the investigation of multineutron haloes. Finally, as the technique of intensity interferometry is applicable to protons, proton-rich nuclei exhibiting similar fewbody clustering may also be explored.

The support provided by the staffs of LPC and GANIL in preparing and executing the experiments is gratefully acknowledged. This work was funded under the auspices of the IN2P3-CNRS (France) and EPSRC (United Kingdom). Additional support from the ALLIANCE program (Ministère des Affaires Etrangères and British Council), the Human Capital and Mobility Program of the European Community (Contract No. CHGE-CT94-0056), and the GDR Noyaux Exotiques (CNRS-CEA) is also acknowledged.
[1] M. Freer et al., Phys. Rev. Lett. 82, 1383 (1999).

[2] P.G. Hansen and B. Jonson, Europhys. Lett. 4, 409 (1987).

[3] M.V. Zhukov et al., Phys. Rep. 231, 151 (1993).

[4] L.V. Chulkov et al., Phys. Rev. Lett. 79, 201 (1997).

[5] M. Zinser et al., Nucl. Phys. A619, 151 (1997).

[6] D. Aleksandrov et al., Nucl. Phys. A633, 234 (1998).

[7] T. Aumann et al., Phys. Rev. C 59, 1252 (1999).

[8] H. Simon et al., Phys. Rev. Lett. 83, 496 (1999).

[9] D. Sackett et al., Phys. Rev. C 48, 118 (1993); K. Ieki et al., ibid. 54, 1589 (1996).

[10] D.H. Boal, C.K. Gelbke, and B.K. Jennings, Rev. Mod. Phys. 62, 553 (1990)

[11] See, for example, D. H. Perkins, Introduction to High Energy Physics (Addison-Wesley, New York, 1987), p. 122.

[12] G.I. Kopylov, Phys. Lett. 50B, 472 (1974).

[13] F.M. Marqués et al., Phys. Lett. B 476, 219 (2000).

[14] R. Lednicky and L. Lyuboshits, Sov. J. Nucl. Phys. 35, 770 (1982).

[15] F. M. Marqués et al. (in preparation).

[16] M. Labiche et al., Phys. Rev. Lett. 86, 600 (2001); M. Lab- iche, Ph.D. thesis, Université de Caen, 1999, LPCC T 99-03.

[17] F.M. Marqués et al., Nucl. Instrum. Methods Phys. Res. A 450, 109 (2000).

[18] K.L. Jones, Ph.D. thesis, University of Surrey, 2001; N.A. Orr, Proceedings of the Tours Symposium on Nuclear Physics, AIP Conf. Proc., 2001 (in press), nucl-ex/0011002.

[19] M. Nikolić, Kinematics and Multiparticle Systems (Gordon and Breach, New York, 1968), p. 33.

[20] E.M. Aitala et al., Phys. Rev. Lett. 86, 770 (2001).

[21] A.V. Belozyorov et al., Nucl. Phys. A636, 419 (1998).

[22] M. Thoennessen, S. Yokoyama, and P.G. Hansen, Phys. Rev. C 63, 014308 (2001).

[23] M.V. Zhukov and B. Jonson, Nucl. Phys. A589, 1 (1995).

[24] P. Descouvemont, Phys. Rev. C 52, 704 (1995).

[25] I.J. Thompson and M.V. Zhukov, Phys. Rev. C 53, 708 (1996).

[26] M. Labiche et al., Phys. Rev. C 60, 027303 (1999).

[27] See, for example, E. Garrido, D.V. Fedorov, and A.S. Jensen, Phys. Lett. B 480, 32 (2000).

[28] P.G. Hansen, Nucl. Phys. A649, 355c (1999). 